BULLETIN Bulletin hispanique

HISPANIQUE Université Michel de Montaigne Bordeaux

117-2 | 2015

Métamorphose(s) : représentations et réécritures

\title{
Métamorphoses ichtyologiques et asines dans la littérature picaresque
}

une échappatoire utopique à la mort sociale ?

\section{Cécile Bertin-Élisabeth}

\section{OpenEdition}

\section{Journals}

Édition électronique

URL : http://journals.openedition.org/bulletinhispanique/4025

DOI : 10.4000/bulletinhispanique.4025

ISSN : 1775-3821

Éditeur

Presses universitaires de Bordeaux

Édition imprimée

Date de publication : 15 décembre 2015

Pagination : 531-548

ISBN : 979-10-300-0041-2

ISSN : 0007-4640

Référence électronique

Cécile Bertin-Élisabeth, « Métamorphoses ichtyologiques et asines dans la littérature picaresque »,

Bulletin hispanique [En ligne], 117-2 | 2015, mis en ligne le 15 décembre 2018, consulté le 05 mai 2019

URL : http://journals.openedition.org/bulletinhispanique/4025; DOI : 10.4000/bulletinhispanique.4025 


\title{
Métamorphoses ichtyologiques et asines dans la littérature picaresque : une échappatoire utopique à la mort sociale?
}

\author{
CÉCIle Bertin-ÉLisabeth \\ Université des Antilles-Guyane
}

Malgré l'apparent réalisme des récits picaresques, diverses métamorphoses - notamment ichtyologiques et asines - y sont privilégiées et mettent en exergue un moment où tout paraît possible. Cette capacité à changer de forme pourrait-elle alors être perçue comme une menace pour l'ordre et exprimer une espérance - utopique - de renaissance sociopolitique?

Mots-clés : Métamorphose, littérature picaresque, renaissance, utopie.

A pesar del aparente realismo de los relatos picarescos, se privilegian diversas metamorfosis -sobre todo ictiológicas y asinescas- que ponen énfasis en un momento durante el cual todo parece posible. ¿Podría representar dicha capacidad en cambiar de forma una amenaza para el orden y podría expresar una esperanza -utópica- de renacimiento sociopolítico?

Palabras-claves: Metamorfosis, picaresca, renacimiento, utopía.

In spite of the apparent realism of rogue's stories, diverse metamorphosis -ichthyological and asine especially- are privileged in them and bring out a moment when all seems possible. Could such ability to change one's form be seen as a threat for the order and express an -utopian- hope for a socio-political rebirth?

Keywords : Metamorphosis, rogue’s literature, rebirth, utopia. 
T E TERME " MÉTAMORPhOSE " qui entre dans les langues vernaculaires Lvia les titres d'Ovide (Les Métamorphoses, 1 ap. J.-C.-8 ap. J.-C. ${ }^{1}$ ) - œuvre omniprésente dans la culture de la Renaissance ${ }^{2}$ - et d'Apulée (Métamorphoses ${ }^{3}$, II ${ }^{\mathrm{e}}$ siècle ap. J.-C.) est un mot qui par ses origines renvoie de façon préférentielle à des sujets mythologiques. Or, malgré l'apparent réalisme des récits picaresques des $\mathrm{XVI}^{\mathrm{e}}$ et $\mathrm{XVII}^{\mathrm{e}}$ siècles, leurs auteurs n'hésitent pas à privilégier les changements d'état et la création de corps hybrides comme on l'analysera pour deux cas récurrents dans la littérature picaresque, à savoir : les métamorphoses ichtyologiques et asines - tout en effleurant le thème de la mue du serpent qui relie ces deux métamorphoses - dont on cherchera notamment les points communs et les divers modes de présentation après avoir questionné la définition et les fonctions de la métamorphose en général.

Il n'empêche que les notions de passage et de frontière inhérentes à toute métamorphose ne sauraient acquérir tout leur sens qu'au regard du devenir convoité. En effet, évoquer de telles métamorphoses physiques ne revient-il pas à inviter à méditer sur l'instant privilégié où tout paraît possible, toute capacité à se métamorphoser pouvant dès lors être perçue comme une menace pour l'ordre, car comme l'affirme Edgar Morin l'espérance éthique et l'espérance politique sont dans la métamorphose. Aussi, ces changements de forme, de nature et de structure en dénonçant pour le moins un manque d'unité - la métamorphose est en soi un instant de crise - ne soulignent-ils pas le statut de monstre social des picaros, descendants de Lazare, et leurs efforts de renaissance socio-politique, soit la transcription dans les récits picaresques d'une poétique de la métamorphose qui intègre tant les apports de l'Antiquité que les nouvelles voie(x)s de l'humanisme ? D'ailleurs, la métaphore corporelle ${ }^{4}$ et de ce fait les métamorphoses du corps n'ont-elles pas toujours constitué une forme de langage privilégié pour traiter de la société et de la question du pouvoir, entre ordre et désordre?

\section{MÉtamorphose(s) : DÉFINITIONS ET FONCTIONS}

Il importe avant tout de convenir d'une définition précise de la métamorphose et des fonctions qui lui sont attribuées. On rappellera que dans les récits de l'Antiquité, les métamorphoses se réalisent suite au caprice d'une divinité. Il

1. Il s'agit d'un poème de plus de 12000 vers qui puise lui-même dans diverses sources dont sans nul doute les Métamorphoses de Nicandre de Colophon. On y voit souvent un recueil de fables étiologiques et anthropogoniques. Cf. Pierre Brunel, Le mythe de la métamorphose, Paris, Armand Colin, 1974, chap. 2 et 3. Il faut attendre en France le XIVe siècle pour que le mot " métamorphose " soit employé, d'abord comme titre des œuvres d'Ovide et d'Apulée. C'est au $\mathrm{XV}^{\mathrm{e}}$ siècle que ce terme devient un mot commun encore fort lié au domaine mythologique. Les dictionnaires en proposent initialement comme définition : " transformation " ou « transmutation ». Ce dernier terme sera ensuite réservé aux choses.

2. Les écoliers y apprenaient le latin.

3. Titre original de cette œuvre de Lucius Apuleus : Asinus aureus.

4. Cf. Georges Balandier, Le détour : pouvoir et modernité, Paris, Fayard, 1985, p. 23-24 : «La métaphore corporelle traduit la logique du vivant en logique du social ». 
peut s'agir également d'une punition pour ceux qui avaient osé affronter les dieux. Platon ne disait-il pas que c'étaient les âmes soumises à la gloutonnerie, à la boisson et à la démesure qui prendraient la forme d'ânes ${ }^{5}$ ?

Chez Ovide, les êtres vivants, une fois installés dans une forme peuvent en changer, sans aucune limitation et s'ensuit alors la création de corps hybrides. En règle générale, les métamorphoses de dieux sont provisoires tandis que celles des hommes tendent à être définitives.

Il appert que lorsque la prégnance mythologique domine, la métamorphose est conçue comme un changement radical (soit le schéma suivant pour la métamorphose mythique : forme initiale, transformation, forme finale) alors que dans d'autres contextes, se voit retenue simplement l'idée de changement, sans véritable modification de la nature d'un être ${ }^{6}$ et sans caractère d'irréversibilité. Ainsi, la métamorphose peut recouvrir des notions aussi diverses que celle du changement complet de l'être ou du simple travestissement vestimentaire, mais on s'en tiendra dans cette étude aux métamorphoses corporelles.

L'une des définitions les plus communes de la métamorphose est donc celle d'un changement de forme, de nature ou de structure qui rend l'être (ou la chose) méconnaissable. Francis Berthelot décompose pour sa part la métamorphose en quatre paramètres ${ }^{7}$ : le sujet qui la subit, l'agent qui la fait subir, le processus selon lequel elle s'accomplit et le produit qui en découle. Quoi qu'il en soit, la métamorphose en tant que changement d'état qui pourrait suspendre le cours du temps préside assurément au mouvement vital ${ }^{8}$ et se retrouve comme mythe au cœur des cosmogonies sacrées. Guy Belzane affirme à cet égard :

[...] le thème de la métamorphose trouve son origine dans les mythes, d'où son ambivalence essentielle : s'y expriment à la fois des aspirations et des terreurs archétypales. Le récit de métamorphose a d'abord pour fonction d'expliquer le monde, de lui donner un sens. Mythe étiologique, il résout à sa manière l'énigme d'un univers inconnu et angoissant, d'une nature étrangère et incertaine?

De surcroît, on retiendra que les métamorphoses appartiennent souvent à la catégorie des obstacles que rencontrent les héros, soit des épreuves à surmonter, lesquelles menacent la liberté du héros et entravent son action. Plutôt qu'une fuite, la métamorphose devient alors un triomphe sur un destin malheureux.

5. Platon, Euvres complètes I, Phédon, Paris, Gallimard, coll. « La Pléiade », p. 801-802, 81 : " [...] ceux dont la gloutonnerie, la démesure, la passion de boire ont été la pratique ordinaire, et qui ne s'en sont pas défendus, c'est vraisemblablement dans des formes d'ânes et d'autres pareilles bêtes que vont se plonger leurs âmes".

6. Voir à ce propos l'analyse d'Hélène Nais, "Pour une notice lexicographique sur le mot "métamorphose" ", dans Poétiques de la métamorphose, Saint-Étienne, Publications de l'Université de Saint-Étienne, 1981, p. 15-25.

7. Francis Berthelot, La métamorphose généralisée : du poème mythologique à la science-fiction, Paris, Nathan, 1993, chap. 1, p. 7-15.

8. On pense au grand branle dont parle Montaigne dans ses Essais, III, 2.

9. Guy Belzane, La métamorphose: Ovide, Perrault, Hugo, Michaux, Paris, Quintette, 1990, p. 8. 
Il est intéressant de remarquer que l'idée de métamorphose (de meta, " qui dépasse ", et morphêe, "forme ") participe aisément de l'esthétique baroque ${ }^{10}$ (et de sa finalité apologétique) avec ses jeux de miroirs et de trompe-l'œil ainsi que sa prédilection pour le mouvant et les effets de dévoilements. Si rien n'est fixe, tout s'avère possible... Déjà les présocratiques développent l'idée de mouvance. À l'intérêt pour la Forme idéale (eidos) platonicienne de nature métaphysique succède celui pour les formes, muables, de nature physique. En affirmant que le propre de la matière est de désirer la forme, Aristote reconnaît que la matière est capable de transformations. Et en permettant de relier métaphysique et physique, ce philosophe dote le changement d'un statut philosophique. L'apport chrétien s'enracine également dans une mythologie de la métamorphose et structure les imaginaires en ce sens, ne serait-ce que parce qu'en Christ, Dieu s'est fait homme.

Assurément, la métamorphose est en soi un instant de crise tout comme le monstre exhibe la crise qui est à son origine ${ }^{11}$. Ajoutons que la métamorphose convoque dans le monde chrétien un aspect sulfureux, en soi subversif, étant donné que ce sont le diable et les sorcières que l'on perçoit communément comme ceux qui la pratiquent.

Ces prolégomènes posés, reste à déterminer quelles sont les métamorphoses retenues dans les récits picaresques et s'il s'agit de métamorphoses lentes ou brutales, réversibles ou permanentes. On ne cherchera pas à construire une sémiotique générale de la métamorphose, mais à s'intéresser à son fonctionnement dans les récits picaresques, entre œuvres modèles et œuvres relais, à partir de l'étude de cas particuliers de métamorphoses physiques.

\section{MÉTAMORPHOSES ICHTYOLOGIQUES ET ASINES}

Deux métamorphoses animales ${ }^{12}$ dominent dans les récits picaresques classiques. On s'intéressera en premier lieu à l'enveloppe ichtyologique dont les

10. Alors qu'à la Renaissance, la métamorphose, le changement, n'est qu’instabilité. Voir par exemple l'analyse de Gisèle Mathieu-Castellani, « Le mythe du phénix et la poétique de la métamorphose dans le lyrisme néo-pétrarquiste et baroque ", dans Poétiques de la métamorphose, op. cit., p. 161-183.

11. Cf. Michel Foucault, Les anormaux : cours au Collège de France (1974-1975), Paris, Le Seuil, 1999.

12. Rappelons la tendance à associer les figures de bouffon à des animaux, notamment ceux que les grands de ce monde appréciaient le plus comme les chiens et les singes. Monique Joly dans La bourle et son interprétation : recherches sur le passage de la facétie au roman (Espagne, XVIXVII siècles), Thèse présentée à l'Université de Montpellier III, juin 1979, Lille, Atelier national de reproduction des thèses, Université de Lille III, 1986, cite à cet égard un extrait significatif : " "Los Monos son los que se precian (de) truhanes, y entretenidos acerca de las personas principales [...]. A vezes los poderosos admitirán estos a su gracia, y despreciarán el trato de los buenos porque anda el mundo al reues" (Gómez Tejada de los Reyes, 1636, Apólogo XVII, p. 101r) ». Estebanillo, bouffon depuis qu'il est entré au service de l'ambassadeur, affirme par la suite : «Aqui fue donde 
exemples les plus probants sont à tirer des secondes parties du Lazarillo ${ }^{13}$ bien que celles-ci aient été longtemps fort décriées (justement souvent à cause de ces métamorphoses $\left.{ }^{14}\right)$, puis quelque peu réhabilitées ${ }^{15}$. François Delpech propose à cet égard une étude très complète où il rappelle l'intérêt pour les origines de ce récit : "On n'a pas manqué enfin de s'interroger sur les "sources", le "genre" et les parallèles littéraires d'un récit dans lequel on veut voir tour à tour, ou tout à la fois, une parodie de la littérature chevaleresque, un apologue sur la vie de Cour, une résurgence du roman de métamorphoses, une satire lucianesque, voire une fable cryptée $»^{16}$.

Il convient de relever dans ces récits l'importance de scènes sous-marines où les protagonistes prennent la forme de thons. Il ressort en effet que pour conjurer la mort, exemplairement mise en exergue dans les scènes de naufrages des seconds Lazarillo, à une époque où l'espace maritime nourrissait de profondes inquiétudes ${ }^{17}$, une renaissance est proposée sous une nouvelle

se me infundió un abismo de gravedad, viendo que de bufón de una Excelencia habia llegado a serlo de una Alteza real; y como otros dan en querer perros, monos, y otros diferentes animales, dio Su Alteza en quererme bien [...], y mostrarlo en mandarme hacer muy ricos y costosos vestidos ", La vida $y$ hechos de Estebanillo González, éd. de Antonio Carreira et Jesús Antonio Cid, Madrid, Cátedra, 1990, II, 8, p. 114. On voit dans ce dernier exemple, les jeux de changements d'apparence par le biais des habits et le recours significatif à une image de métamorphose animale, celle du poisson pour exprimer combien il se sent à l'aise : "Gustaba de llevarme a la caza [...] y a dar alegría a sus súbditos y regocijo a la corte; en cuyo apacible estruendo y sonoroso ruido me hallaba como el peje en el agua $[\ldots]$ ", p. 115.

13. Miguel de Cervantès associe monde de type picaresque et madrague dans La ilustre fregona et va jusqu'à mettre en parallèle l'illustre laveuse et les thons. Cf. l'article de Araceli Guillaume-Alonso, «La séduction de la madrague : réalité sociale et technique littéraire dans le récit de Cervantès ", dans Pierre Civil (coord.), Écriture, pouvoir et société en Espagne aux XVI et XVII siècles, Hommage du CRES à Augustin Redondo, Paris, Publications de la Sorbonne, Presses de la Sorbonne Nouvelle, 2001, p. 425-432.

14. On citera en guise d'exemple l'étude de José María Cossío qui y voit une œuvre écrite à la va-vite suite au succès du premier Lazarillo; la transformation en thon lui paraissant de surcroît absurde, "Las continuaciones del Lazarillo de Tormes ", Revista de Filología Española, $1941, n^{\circ} 25$, p. 514-523.

15. Cf. R. Zwez (qui propose de voir le roi des thons à l'image de Charles Quint), Hacia una revalorización de la Segunda Parte de Lazarillo (1555), Madrid-Valence, Albatros, 1970 ; Pierina E. Beckman, El valor literario del Lázaro de 1555. Género, evolución y metamorfosis, New York, Peter Lang, 1991 ; François Delpech, "Lazare, l'eau, le vin et les thons. Éléments pour une recherche sur les corrélats folkloriques de la première continuation du Lazarillo (Anvers, 1555) ", dans Pierre Civil (coord.), Écriture, pouvoir et société en Espagne aux XVI et XVII siècles, op. cit., p. 309-327 et Valentín Núnez Rivera, "Claves para el segundo Lazarillo, 1555. El continuador anónimo interpreta su modelo ", Bulletin hispanique, $\mathrm{n}^{\circ}$ 2, déc. 2003, Université Michel de Montaigne, Editions Bière, p. 333-369.

16. François Delpech, art. cit., p. 309-310.

17. Estebanillo confirme cette peur de la rigueur des flots, Estebanillo González, éd. cit., II, 13, p. 352 : "Aqui fue donde de todo punto aborrecí el agua, y adonde acabé de confirmar por insensatos a los hombres que pueden caminar por tierra, comiendo cuando quieren y bebiendo cuando gustan, y se ponen a la inclemencia de los vientos, al rigor de las ondas, a la fiereza de los piratas, y finalmente ponen sus vidas en la confianza de una débil tabla, sin considerar el peligro de un escollo, el 
forme : ichtyologique (où l'honneur est possible ${ }^{18}$ ). La mer convoque en effet à la fois l'idée de séparation (dilation) d'avec les hommes et de lien (relation) ${ }^{19}$ avec Dieu et sa présence renforce de fait les images de continuité/discontinuité propres à toute métamorphose.

Toutefois, la fonction (valeur fonctionnelle, symbolique, politique...) de ces scènes sous-marines - pour des picaros au programmatique nom de Lazare le

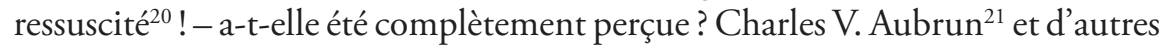
critiques proposent d'interpréter ces thons comme étant une représentation des Turcs. Certains critiquent l'ingratitude des Grands de ce monde et les préoccupations courtisanes; d'autres encore s'interrogent quant à la présence de la Vérité comme clé de lecture. Cette dernière reproche justement à Lazare d'avoir oublié les promesses faites sous l'eau une fois revenu sur terre et lui inflige le châtiment suivant : «[...] haz libro nuevo $»^{22}$. On s'arrêtera également sur l'interprétation de Máximo Saludo Stephan ${ }^{23}$ qui considère qu'il importe de lire la rencontre de Lazare avec la Vérité comme une rencontre avec le Christ ; suggérant de surcroît que la roche où ils se retrouvent n'est autre que l'île de Malte (à la croix formée de quatre queues de thon ${ }^{24} \ldots$...

De l'arrière-plan traditionnel - traditions quasi para-mythologiques - on retiendra en premier lieu la possible réminiscence de la légende du "Peje Nicolao $»^{25}$. En effet, diverses légendes du XII ${ }^{e}$ au XVII ${ }^{e}$ siècle évoquent le

riesgo de una sirte y el daño de un bajio, el temor de un banco, el sobresalto de una playa y la soberbia de una bestia fiera y indómita, y que le basta ser mujer para ser mudable y voltaria".

18. Lazare entre dans le monde aristocratique sous-marin. François Delpech parle à juste titre, vu l'idéalisation héroïque, d'" intermède épico-ichtyologique " (art. cit., p. 313).

19. Les Grecs utilisaient pour désigner la mer à la fois les termes thalassos, pelagos et pontos (en latin pontis et en français " pont»), soit pour cette dernière acception l'idée que la mer unit deux éléments.

20. Cf. mon article "Approche diachronique de la problématique du prénom "Lazare" dans la littérature espagnole $\left(\mathrm{XVI}^{\mathrm{e}}-\mathrm{XX}\right.$ e siècle) " présenté au colloque du CRLMC organisé à l'université Blaise Pascal-Clermont II les 4-5 et 6 décembre 2006 : Figures de Lazare dans le domaine russe et au-delà coordonné par Régis Gayraud. En cours de publication.

21. Charles V. Aubrun, "La dispute de l'eau et du vin ", Bulletin hispanique, 1957, LIX, p. $453-456$.

22. Segunda parte de Lazarillo de Tormes (1555), La novela picaresca española, éd. de Florencio Sevilla Arroyo, Madrid, Castalia, 2001, chap. XVII, p. 42. La citation complète est : «- Tú, Lázaro, no te quieres castigar: prometiste en la mar de no me apartar de ti, desque saliste casi nunca más me miraste. Por lo cual la divina justicia te ha querido castigar, y que en tu tierra y en tu casa no halles conocimiento, mas que te vieses puesto como malhechor a cuestión de tormento. Mañana vendrá tu mujer y saldrás de aqui con honra, y de hoy más, haz libro nuevo".

23. Máximo Saludo Stephan, Misteriosas andanzas atunescas de "Lázaro de Tormes». Descifradas de los seudo-jeroglificos del Renacimiento, Saint-Sébastien, Izarra, 1969, p. 15 : "Lázaro encontró a la Verdad refugiada en una roca en el mar, es decir el mismo Cristo. Y podemos ya, anticipando los resultados de este estudio sugerir que la roca, refugio de Cristo, será la isla de Malta ". Rappelons que selon la tradition, l'île de Malte servit de refuge à saint Paul et est assimilée à la baleine de Jonas !

24. Ibid., p. 26.

25. Cf. Julio Caro Baroja, "El 'Pesce Cola' o el 'Peje Nicolao' ", Revista de dialectología y tradiciones populares, Madrid, Instituto Miguel de Cervantes, Consejo Superior de Investigaciones Científicas, 1984, n³9, p. 7-16. 
"Peje Nicolao " dans différentes zones méditerranéennes, à Naples, Messine ou encore à propos de la côte de Cadix ${ }^{26}$. Cet homme-poisson aurait subi une malédiction maternelle, suite à l'irritation causée par son goût pour les flots. Il lui aurait été possible de surcroît de s'introduire dans le corps de très gros poissons, soit la reprise du thème de Jonas. En second lieu, il convient de souligner le lien avec le duc de Medina Sidonia dont la famille possède le monopole de la pêche au thon, les fameuses "almadrabas ${ }^{27}$. Ce monde de la pêcherie a mauvaise réputation et apparaît décrit comme "un haut lieu de la canaille ${ }^{28}$, proche du monde picaresque ${ }^{29}$. La satire dans un contexte de rivalité politique quant à cette prestigieuse famille et ses origines, mêlée aux réminiscences folkloriques ${ }^{30}$, semble proche du protagoniste-narrateur picaresque qui, se retrouvant dans un monde inversé, inverse à son tour nos perceptions... Il serait pour le moins erroné d'y voir une pure fantaisie. On ne peut également qu'être conscient que le thème de la métamorphose en animal marin fut parfois interprété comme un châtiment divin, suite à une transgression ${ }^{31}$. Et les picaros ne sont-ils pas présentés justement réalisant diverses actions qui tendent à transgresser l'ordre social ?

Dans la Segunda parte de Lazarillo de Tormes y de sus fortunas y adversidades, œuvre anonyme de 1555 publiée à Anvers, Lazare à qui la vie sourit désormais souhaite encore une meilleure fortune et s'embarque - encouragé par sa femme et maittresse de l'archiprêtre... - pour " Argel » afin de combattre les Maures. Il n'arrive pas à bon port, suite à une violente tempête. Terrorisé par la perspective du naufrage annoncé, ce picaro s'enivre sans retenue ${ }^{32}$. Et lorsqu'il se voit englouti par les eaux et prêt à être dévoré par les thons, Lazare se transforme lui aussi en thon ${ }^{33}$. En somme, mort en quelque sorte à la vie humaine, Lazare renaît sous la forme d'un poisson, vivant désormais comme l'un d'eux. La renaissance ne se réalise pas uniquement sur le plan physique mais aussi du point de vue moral puisque sous l'eau ce Lazare-poisson se comporte comme

26. Cervantès évoque cette légende dans le Quichotte, II, chap. XVIII. Don Quichotte parle des qualités de tout bon chevalier errant et précise : « [...] digo que ha de saber nadar como dicen que nadaba el peje Nicolás, o Nicolao [...]".

27. Cf. Araceli Guillaume Alonso, «Autour des confréries de marginaux : les Almadrabas au Siècle d'Or ", dans A. Redondo (éd.), Les parentés fictives en Espagne (XVI $-X V I I^{\mathrm{e}}$ siècles), Paris, Publications de la Sorbonne, 1988, p. 135-143.

28. Ibid., p. 135. Araceli Guillaume Alonso indique même qu’à certaines périodes, la Maison de Medina Sidonia allait recruter ses «ventureros » dans la prison de Séville (ibid., p. 139).

29. Araceli Guillaume Alonso cite un contemporain : "es gente ruin y picara " (ibid.).

30. François Delpech attire notre attention sur les croyances en des personnages fantastiques: les " Nûtons " ou "Lûtons ", entités souvent diaboliques, " liées aux eaux, aux chevaux (voir chapitre suivant), à la fonction de guide, à l'obscurité et à la cécité (ou autres problèmes de vision), aux trésors cachés, aux métamorphoses animales, à la Canicule [...] ", art. cit., p. 324.

31. Cf. François Delpech, art. cit., p. 321.

32. Segunda parte de Lazarillo de Tormes, op. cit., p. 24.

33. Rappelons que J. B. de Salazar dans son Historia de la Isla de Cádiz (1610) souligne que le thon équivaut au porc sur terre. Cf. Máximo Saludo Stephan, op. cit., p. 67 : «En el modo de pensar de entonces, el atún, puerco marino, habia llegado a tomar un sentido despectivo, simbolo de lujuria como el puerco, de cobardía, de poca fe: huía de la sal de la fe "; voir également la note 52. 
un véritable gentilhomme alors que sur terre il ne pratique que le mensonge. Toutefois, ce protagoniste retrouve sous l'eau, chez d'autres personnages, les déviances et les corruptions pratiquées sur terre. Ainsi, son ami Licio mandaté par le roi des thons lui propose en mariage l'ancienne maitresse du roi, Luna, d'où le commentaire de Lazare : " [...] a subir acierto: razón es de arcipreste a rey haber salto $"^{34}$.

Cette période noble de sa vie s'arrête brutalement alors qu'il accompagne les femelles thons frayer et que des pêcheurs peu regardants le hissent hors de l'eau, lui arrachant une partie de son enveloppe animale. Revenu sur terre, doté désormais d'un corps gémellaire, mi-homme mi-thon ${ }^{35}$, Lazare est transformé en monstrueux poisson de foire par ces pêcheurs sans scrupules. Sa partie de poisson se putréfie peu à peu, annonçant par là même une mort possible. Ayant réussi à se libérer de son enveloppe et de ses geôliers, Lazare retourne à Tolède, quatre années après son départ (à l'instar du Lazare biblique qui avait été ressuscité alors qu'il était mort depuis quatre jours...), où l'on ne le reconnaît point tant la métamorphose antérieure a laissé de traces. Pas de retour possible donc à l'espace de départ, d'où son choix de se rendre à Salamanque où il brille par ses joutes oratoires à l'université.

Mais pourquoi une transformation en poisson et plus précisément en thon, les antécédents littéraires les plus connus étant celui du coq dans Le coq de Lucien - qui inspire indéniablement le Crotalón de Cristóbal de Villalón ${ }^{36}$ - et de l'âne chez Apulée ? Notons que l'ami de Lazare-thon se prénomme Licio alors que le protagoniste de l'Âne dor porte le nom fort proche de Lucio. En revanche, on relève une divergence apparente : alors que chez ces prédécesseurs, le métamorphosé vit parmi les hommes, dans la Seconde partie du Lazarillo de 1555, Lazare-thon partage la vie des thons. Cependant, les remarques dont il émaille sa description de la vie sous-marine se réferent constamment à la vie sur terre. Lucio, après avoir repris sa forme humaine reste au service d'Isis qui l'aide à recouvrer sa forme première et Lazare promet pour sa part à la Vérité de ne plus l'oublier. Covarrubias nous invite quant à lui à considérer que les thons sont les plus voraces et donc les plus cruels des poissons, soit une adaptation de "l'homme est un loup pour l'homme" en "l'homme est un thon pour l'homme »!

On aura sans doute relevé le lien entre l'image du poisson et le sens grec de l'ichtus-INRI ${ }^{37}$ : le poisson-symbole des premiers chrétiens qui est utilisé

34. Segunda parte del Lazarillo (1555), op. cit., chap. XIV, p. 39.

35. Michel Foucault parle de "métamorphose verticale " quand se joignent ainsi deux êtres (qu'il oppose à la "métamorphose horizontale » quand il y a changement de forme, l'une à la suite de l'autre), Raymond Roussel, Paris, Gallimard, 1963.

36. Cristóbal de Villalón, El Crotalón, éd. de Augusto Cortina, Madrid, Espasa-Calpe, 1973. Il se transforme également en porc.

37. Rappelons les sens religieux des termes Ichtus (Jésus, Christ, Dieu, Fils, Sauveur) et INRI (Jésus de Nazareth Roi des Juifs). Ajoutons à ces symboles chrétiens la croix dont il importe de préciser qu'elle symbolise aussi la jonction des deux mers. Or, l'élément maritime joue un rôle important dans les secondes parties des Lazarillo. 
de fait comme un symbole de résurrection. Le choix de cette métamorphose sous-marine acquiert donc un sens loin d'être anodin. Les contemporains du premier Lazarillo ont-ils été sensibles à l'association entre le Lazare biblique et le fils de Dieu ? Cette image d'un Lazare-poisson sera reprise en 1620 par Juan de Luna, auteur protestant, qui propose une nouvelle seconde partie : Segunda parte de la vida de Lazarillo de Tormes, sacada de las crónicas antiguas de Toledo, publiée à Paris, loin de la censure inquisitoriale. Il y est de nouveau question d'un voyage à "Argel " et d'un naufrage ainsi que du vin comme élément salvateur. Au fond des flots, Lazare trouve un coffre rempli d'or et de joyaux qu'il s'attache aux pieds en vue de le remonter sur le rivage et d'assurer sa fortune. Toutefois, arrivé à la surface, des pêcheurs le transforment en monstre marin qu'ils exhibent de ville en ville. Un jour, à Tolède, ce Lazare-poisson revoit sa femme, enceinte des œuvres de l'archiprêtre et manque d'en mourir, en se noyant quasiment dans l'eau de l'infâme bassin où il croupit. Comme dans l'autre seconde partie du Lazarillo, l'étape de reconnaissance s'avère malaisée. Une fois son identité reconnue, Lazare engage un procès contre l'archiprêtre. Cette démarche lui sourit tant que durent ses finances, ce qui souligne encore le difficile retour à un état premier.

L'élément liquide (maritime), lieu de vérité, sous-tend toute la problématique de la métamorphose annoncée par la naissance-baptême dans les eaux du Tormes. On comprend mieux dès lors les comparaisons que propose le Guzmán avec le prophète Jonas qui refusa d'obéir à Dieu et qui traitent en fait du problème de la conversion, que ce soit dans la narration d'Ozmin y Daraja $a^{38}$ ou lors de l'épisode de la galère ${ }^{39}$, ventre métaphorique de la baleine du Livre de Jonas. Or, dans l'art roman et gothique, la baleine de l'épisode de Jonas peut symboliser la préfiguration de la mort du Christ et de sa résurrection ${ }^{40}$. En somme, on renoue avec le désir d'universalisme du livre de Jonas. On trouve à nouveau cette référence à Jonas et à la baleine dans la Seconde partie du Lazarillo de Juan de Luna où l'on assiste à une métamorphose maquillée :

Cuando el buen Lázaro estaba dando gracias a Dios por haberle sacado del vientre de la ballena [...], tomáronme entre cuatro de aquéllos, que parecían más verdugos de los que crucificaron a Cristo que hombres; atáronme las manos y pusieron una barba y casquete de moho, sin olvidar los mostachos, que parecía salvaje de jardin; vime como trucha montañesa ${ }^{41}$.

38. Voir l'analyse de M. Cavillac à ce propos, Gueux et marchands dans le Guzmán de Alfarache (1599-1604), Bordeaux, Institut d'Études ibériques et ibéro-américaines de l'Université de Bordeaux, 1983, p. 122.

39. Estebanillo, après le vol du chevreau, va dormir symboliquement dans la rue de la Galère ; du nom d'une maison correctionnelle pour femmes : " [...] fuime a dormir a la calle de la Galera, donde de ordinario hospedan la gente de mi porte ", Estebanillo González, op. cit., I, 4, p. 201-202.

40. Dans cet ouvrage, un châtiment est prévu avec une "espina de ballena " où Máximo Saludo Stephan lit une allégorie de la justice de Malte, op. cit., p. 33. Plus loin, p. 77, il propose l'analyse suivante : "La Verdad, expulsada de la tierra [...] puede ser una de las variaciones de la leyenda de Jonás".

41. Juan de Luna, Segunda parte de la vida de Lazarillo de Tormes, sacada de las crónicas antiguas de Toledo (1620), éd. de Florencio Sevilla Arroyo, Madrid, Castalia, 2001, chap. IV, p. 809. 
On l'a déjà suggéré, ce ne sont d'ailleurs pas les seules œuvres de cette période à s'intéresser aux transformations. Le Crotalón, anthologie de divers textes et le Diálogo de las transformaciones convoquent l'idée de métamorphose et en font une thématique propre à cette époque. De même, dans El coloquio de los perros de Cervantès deux chiens sont dotés du divin don de la parole, comme une autre perspective de métamorphose. Assurément, cette présence du merveilleux dans un genre qui privilégie certes le réalisme demeure bien visible dans les secondes parties du Lazarillo.

Mais le recours à la métamorphose - et donc au franchissement des limites s'exprime-t-il toujours de la même façon dans ces récits ? De la figure du poisson métaphore christique, l'on passe souvent dans les récits picaresques au recours aux équidés et notamment à l'âne. L'âne est présenté de façon favorable dans l'Ancien et le Nouveau Testament. Il se trouve de surcroît présent dans la crèche ${ }^{42}$ et assiste donc à la naissance du Christ qu'il sauve d'une mort certaine en l'emmenant en Égypte, comme le Christ sauve Lazare de la mort.

Le glissement du poisson à l'âne n'est pas malaiséà comprendre si l'on remonte à la très ancienne association, présente dans de nombreux mythes, entre l'âne, l'eau et l'immortalité, mise remarquablement en exergue par Waldemar Deonna dans "Laus Asini. L'âne, le serpent, l'eau et l'immortalité " ${ }^{43}$. On s'inspirera généreusement des connaissances mythologiques et historiques de cet auteur dans les lignes qui vont suivre. Ainsi ce critique indique l'existence d'une monnaie de Cyzique associant l'âne et le thon ${ }^{44}$ et synthétise le symbolisme de l'âne de la façon suivante : "Intimement lié aux eaux, des sources, des fontaines, des fleuves, même de la mer, il est un génie des eaux, qui donnent la vie et la mort $"{ }^{45}$. On ajoutera que dans le monde grec, Athéna Hippia (fille de Mètis) est étroitement associée à Poséidon Hippios (lié à Pégase), avec qui elle partage un domaine commun : celui du cheval. La pensée grecque insiste d'ailleurs sur les affinités entre navire et cheval ${ }^{46}$.

Parmi les symboles de renaissance, l'homme dans sa crainte du néant définitif - l'utopie de l'immortalité n'est-elle pas communément partagée chez les hommes? - s'est intéressé notamment aux animaux qui changent de peau (peau et corps étant alors vus comme des équivalents, concevant cette transformation

42. Même si c'est un symbolisme récent. Notons que sur certains graffiti anti-chrétiens retrouvés à Rome, on peut observer des ânes crucifiés avec même parfois des légendes explicites quant à l'incompréhension du culte chrétien puisque ces derniers sont accusés d'adorer l'animal le plus vil : l'âne.

43. Waldemar Deonna, "Laus Asini. L'âne, le serpent, l'eau et l'immortalité ", Revue belge de philosophie et d'histoire, 1956, XXXIV, $1^{\mathrm{re}}$ (p. 5-42), $2^{\mathrm{e}}$ (p. 337-364) et $3^{\mathrm{e}}$ partie (p. 623-658).

44. Ibid., p. 43, note 4. Máximo Saludo Stephan, op. cit., p. 66, affirme : "Aquellas primeras costumbres religiosas del culto de Osiris, llegadas a Sevilla con Hércules, iban codificadas en misteriosos libros sagrados escritos con jeroglíficos. Lo poco que se sabia de ellos venía también de unas lineas del "Asno de oro". En un comentario latino de Apuleyo, salido a luz en Venecia (1504), Felipe Beroaldo decía que esta escritura imitaba las escamas de los peces".

45. Waldemar Deonna, art. cit., $3^{\mathrm{e}}$ partie, p. 636.

46. Marcel Detienne et Jean-Pierre Vernant, Les ruses de l'intelligence : La mètis des Grecs, Paris, Flammarion, coll. «Champs », 1974, p. 224. 
comme l'accès à une nouvelle vie. Or, il existe un lien entre le serpent et l'âne ${ }^{47}$ dans divers contes d'ânes et de serpents, relevés d'ailleurs par Voltaire ${ }^{48}$ dans l'introduction de son Essai sur les mours. Il s'agit toujours d'un âne assoiffé qui remet au serpent sa charge - recette contre la vieillesse donnée aux hommes pour pouvoir se désaltérer, le serpent acquérant ainsi l'immortalité. On relève d'ores et déjà l'omniprésence de la soif ${ }^{49}$ contractée par l'âne, que l'on retrouve sous une autre forme chez le picaro : soif d'élévation sociale, soif d'utopie en somme, car l'idée de soif qui transcende l'aspect physique est en soi aspiration à une vie nouvelle. Le drame se noue indéniablement autour d'une source aqueuse. Et, en effet, l'eau et l'immortalité se sont vues de tous temps associées ${ }^{50}$.

Le fait que l'âne aille de pair avec les liquides - dont le vin de par son association avec Dionysos - convoque de surcroît l'idée de fécondités ${ }^{1}$. Il se voit donc associé à la naissance et à la renaissance vu son lien avec l'immortalité évoqué précédemment. Lâne est également libido ${ }^{52}$, élément instinctif, tantôt bienfaisant ${ }^{53}$, tantôt malfaisant, noble ou méprisé. L'Église chrétienne en fait même le symbole du peuple juif qui n'a pas connu la parole "salvatrice " ${ }^{54}$. On comprend mieux alors comment les convertis de Juifs - dont les picaros... purent être associés à ces animaux.

La liste serait longue des occurrences grecques. Retenons quant à nous Lamia, déesse marine, fille de Poséidon. Il semble intéressant de relever qu'en espagnol ce terme désigne outre une espèce de monstre femelle, une prostituée, ce qui relie le vocable «lamia » avec le monde des marginaux. Et le Diccionario de Autoridades détaille : "pescado cetáceo de desmesurada grandeza [...]. Ha sucedido hallarse en el vientre de una Làmia un hombre entero con su lóriga y arnés:

47. Waldemar Deonna y voit des ressemblances avec la légende de Gilgamesh.

48. Voltaire évoque des fables asiatiques alors que W. Deonna déclare que la source de Voltaire est Nicandre de Colophon (fin du III ${ }^{e}$ s. après J.-C.) ou Élien (Aelien) de Préneste (II ${ }^{e}$ s. après J.-C.) et qu'elle se rattache au mythe de Prométhée. Zeus aurait donné cette recette contre la vieillesse aux hommes pour les remercier de lui avoir indiqué le vol du feu par Prométhée. Le lien avec Prométhée ne doit pas nous surprendre étant donné que l'eau et le feu sont associés de façon récurrente dans les diverses croyances.

49. Cette soif est directement liée aux serpents, notamment la dipsade dont la morsure fait naitre une soif que rien ne peut apaiser. Cf. W. Deonna, art. cit., $2^{\mathrm{e}}$ partie, p. 338-340.

50. Ainsi Gilgamesh cherche la plante d'immortalité au fond de la mer.

51. Comme le scorpion à qui il se trouve aussi associé.

52. Feu et eau vont souvent de pair. On rappellera la relation entre le feu et la sexualité. Cette dimension sexuelle de l'âne est présente chez Francisco Delicado dans La Lozana andaluza, éd. de Carla Perugini, Séville, Fundación José Manuel Lara, 2004, chap. VII, p. 42.

53. W. Deonna rappelle que les Israélites furent sauvés dans le désert par des ânes qui leur indiquèrent des sources, art. cit., $2^{\mathrm{e}}$ partie, p. 352. Il ajoute, p. 363 : «L'âne est exempté de la loi qui sacrifie à l'Éternel tous les premiers nés, hommes et animaux; il est racheté par l'offrande d'un agneau ou d'un chevreau, tout comme le premier-né des hommes est racheté ; ceci en souvenir de la captivité en Égypte, alors que l'Éternel tua tout premier-né des Égyptiens, homme et bête, mais, pour ce qui concerne l'âne, assurément en mémoire du service qu'il a rendu dans le désert ; cette exception confirme sa valeur sacrée ». On attribue de surcroît à l'âne diverses vertus prophylactiques.

54. W. Deonna, art. cit., $3^{\mathrm{e}}$ partie, p. 654. 
y por esta causa entienden algunos Autores haber sido Làmia la que tragó al Profeta Jonás " ${ }^{55}$ (María Moliner parle plutôt d'une sorte de requin ${ }^{56}$ ). Le dictionnaire Furetière (1690) corrobore l'idée que l'on aurait trouvé des hommes entiers, de surcroît armés, dans les ventres de ces poissons gigantesques et que certains croyaient que ce fut ce poisson qui dévora Jonas et non pas une baleine ${ }^{57}$.

On retrouve, désormais sans surprise vu l'existence de liens étroits entre les poissons issus de l'eau et les ânes, l'image de Jonas et du monstre marin si liée à celle du picaro. Indéniablement, sur un mode burlesque voire subversif, cette figure de l'âne parcourt la littérature picaresque depuis le Lazarillo ${ }^{58}$ matriciel où le protagoniste doté d'un père qui fut "acemilero » se fait porteur d'eau, métiers directement liés à l'âne et à l'élément liquide ! À l'influence de la traduction en castillan de L'âne dor ou les métamorphoses d'Apulée ${ }^{59}$ par Diego López de Cortegana $^{60}$ s'ajoute celle de la tradition folklorique. C'est d'abord l'âne qui porte l'eau que vend Lazare, étape-clé - «Este fue el primer escalón que yo subi para venir a alcanzar buena vida [...] ${ }^{61}$ - que l'on ne découvre qu'à la toute fin de cet épisode, une fois la transformation vestimentaire acquise : «Desque me vi en hábito de hombre de bien, dije a mi amo se tomase su asno, que no quería más seguir aquel oficio " ${ }^{62}$. L'eau préfigure la renaissance et l'âne, porteur du fils de Dieu, se convertit à sa manière en l'annonciateur d'une nouvelle vie possible. Mais, travaillant avec un alguazil, Lazare trouve trop dangereux de faire régner la Justice et préfère vendre du vin ; choix symbolique de celui qui se détourne de la voie possible du Bien pour celle, plus aisée et non honorable, des plaisirs dionysiens (vin et femme).

Pour Guzmán, cette rencontre se fait sur le mode de l'onophagie, cette fois-ci dès le début de ses mésaventures (I, I, 5) et non pas en fin d'ouvrage comme pour son prédécesseur. À la suite de cet épisode, la Justice intervient contre l'aubergiste à l'origine de la tromperie. Monique Joly note que l'onophagie ${ }^{63}$ a pu être assimilée au fratricide de la Genèse et rappelle ce véritable tabou alimentaire qui

55. On a modernisé la graphie.

56. Comme les dictionnaires français modernes. Le Larousse précise qu'il s'agit d'un vivipare dont les embryons incubent dans l'utérus et se nourrissent des œufs non fécondés.

57. Furetière : « [...] Quelques-uns ont crû que ce fut ce poisson qui dévora Jonas, et non pas une baleine, à cause que les Païens ont feint qu'Hercule avait demeuré trois jours dans le ventre du canis carcharias, qui est un autre nom qu'on a donné à ce poisson. Les lamies sont autrement appelées chiens de mer".

58. On la retrouvait déjà dans La lozana andaluza qui cite même Apulée (F. Delicado, op. cit., chap. LXV, p. 341).

59. Apulée amplifie le thème déjà traité par Lucien. Il est à noter qu'Apulée choisit une cérémonie isiaque pour que son protagoniste recouvre sa forme primitive. Or Isis n'était-elle pas liée à la fécondité comme Cybèle ? Rappelons que Lucius voulait se transformer en hibou et se retrouve finalement avec le corps d'un âne.

60. Fonger de Haan indique à propos de cette traduction dans "Pícaros y ganapanes ", Homenaje a Menéndez y Pelayo, Madrid, Librería General de Victoriano Suárez, 1899, t. II, p. 149-190, (p. 151, note 4) : «Primera edición, sin fecha ni lugar, 1513, en folio [...]».

61. Lazarillo, op. cit., $\sigma^{\mathrm{e}}$ traité, p. 126.

62. Ibid., p. 127.

63. Monique Joly, op. cit., p. 517. 
voulait que l'homme qui mange la chair d'une bête de somme devienne comme cet animal et se voie de ce fait traité en bête de somme ${ }^{64}$, soit une ingestion monstrueuse de viande interdite ${ }^{65}$ pour un animal lui-même monstrueux étant donné que le mulet est issu du croisement de deux espèces différentes et est stérile $^{66}$. Indéniablement, la crainte de l'ingurgitation d'une nourriture impure se révèle comme pulsion meurtrière de dévoration de l'Autre. Elle agit dans les récits picaresques comme moment de re-connaissance de son abjection par le picaro. Ce thème accompagne donc le mouvement d'intériorisation de l'abject, de l'anomie ${ }^{67}$ (a-nomie - nomos -, soit la transgression de la juridiction divine et/ou humaine).

Le picaro est alors le plus souvent l'objet d'une mystification de la part d'aubergistes fraudeurs, sauf dans le cas d'Estebanillo qui se transforme certes en hôte, mais offre de la viande de cheval et non point d'âne ou de mulet au caractère infamant. Quant à Pablos, c'est également au début de son récit lors $\mathrm{du}$ fameux épisode carnavalesque du "rey de gallos" (I, 2), que se déroule sa rencontre avec un équidé. Ici point d'âne stricto sensu ${ }^{68}$, mais un cheval qui n'en est pas un, un anti-cheval (quasiment monstrueux) réduit à l'état de rosse, soit une dégradation supplémentaire pour un équidé construit et déconstruit :

[...] salí en un caballo ético y mustio, el cual, más de manco que de bien criado, iba haciendo reverencias. Las ancas eran de mona, muy sin cola; el pescuezo, de camello y más largo; tuerto de un ojo y ciego del otro; en cuanto a edad, no le faltaba para cerrar sino los ojos; al fin, él más parecía caballete de tejado que caballo, pues, a tener una guadaña, pareciera la muerte de los rocines [...] pareciera un cofre vivo ${ }^{69}$.

64. Ainsi, il n’est pas anodin, lorsque Guzmán découvre la dépouille du mulet, qu'il indique qu'il manque (et donc qu'il avait ingéré...) la cervelle et la langue.

65. Monique Joly, op. cit., p. 497.

66. Rappelons à cet égard l'origine animale de nombreux termes pour désigner les métis, associés de fait dans un premier temps à des monstres. Cf. Roger Toumson, Mythologies $d u$ métissage, Paris, PUF, 1998, p. 88 : «Quand il apparaît en français au XIII siècle, le mot "mestis" signifie "qui est fait moitié d'une chose, moitié d'une autre". Son champ référentiel est d'abord animalier. Au sens immédiat le mot désigne un animal engendré de deux espèces et qui n'est donc pas de race pure [...] Ce sens s'applique prioritairement à la race canine comme l'indique la locution "chien mestis". C'est encore en ce sens propre que le mot apparait chez Buffon, au tome 2 de l'Histoire naturelle. L'usage du mot ayant été étendu aux êtres humains, deux champs référentiels se sont superposés, le champ référentiel animalier demeurant toutefois prédominant ». Roger Toumson cite alors Furetière pour la définition du mulet, p. 91 : «Bête de somme engendrée d'un âne et d'une cavale ou d'un cheval et d'une ânelle. Les mulets n'engendrent point, parce qu'ils viennent de différentes espèces, comme les monstres ».

67. L'Eucharistie est alors la catharsis de ce phantasme de dévoration. Le corps sans péché du Christ est rédempteur de tous les péchés.

68. Dès l'Antiquité, l'âne a parfois été considéré comme une monture infamante et le fait de le chevaucher peut constituer un châtiment, surtout lorsque le condamné avait la tête du côté de la queue de l'âne, comme par cet usage de l'envers pour défaire le mal qui avait été fait. Cf. W. Deonna, art. cit., $3^{\mathrm{e}}$ partie, p. 641-642. Cet auteur remarque que ce fut souvent la punition des femmes adultères ou prostituées.

69. Francisco de Quevedo, El Buscón, éd. de Domingo Ynduráin, Madrid, Cátedra, 1995, I, 2, p. 110. 
Ce cheval paraît en cours de métamorphose, à la fois mort et vivant. Son corps de "cofre vivo" annonce de façon symbolique la tombe (sociale) de ce protagoniste qui va s'enfoncer au propre et au figuré dans l'immonde boue excrémentielle ${ }^{70}$ d'un marché populeux et populaire. On comprend peut-être mieux avec ce regard neuf, la phrase qui fait suite à la description de cette rosse : "Yendo, pues, en él, dando vuelcos a un lado y otro como fariseo en paso [...] $»^{71}$. Il convient sans doute de lier ce rappel d'une procession et de majesté ${ }^{72}$ du Christ, dont la figure inversée (qui fait d'un jeune âne un vieux cheval) est ici Pablos, «fils» (descendant) de Lazare. Ce n'est pas la mort du Christ qui se voit préfigurée ici, mais bien celle des projets de "caballero » de Pablos, celle de son désir d'ascension sociale. Et c'est encore monté sur un équidé ${ }^{73}$ - en une possible association avec le Quichotte et la mule de Sancho : "Yo iba caballero en el rucio de la Mancha ${ }^{74}$ - que Pablos, qui veut changer de tenue vestimentaire pour se rendre à la Cour, fait la rencontre de don Toribio. Pas étonnant que don Toribio veuille monter sur cet équidé. Et il y parvient symboliquement avec l'aide de Pablos: "húbelo yo de subir " ${ }^{75}$. Cette élévation semble en somme à comprendre comme une réunion dans la dégradation. De plus, pour soigner leur apparence, l'une des règles des picaros avec qui s'acoquine Pablos à Madrid est la suivante: "Estamos obligados a andar a caballo una vez cada mes, aunque sea en pollino, por las calles públicas [...] $»^{76}$.

Quant à Estebanillo, il parle d'entrée de jeu de lui-même comme d'une sorte d'équidé monstrueux ("centauro a lo picaro, medio hombre y medio rocín " ${ }^{77}$ ) et se compare également à un "pollino sardesco " ${ }^{78}$. Il se fait même "merchante de hierros y clavos de herrar caballos ${ }^{79}$, devenant le cuisinier de don Pedro de Ulloa, " capitán de caballos ${ }^{80}$ et propose à ses compagnons de la soldatesque des pâtés de cheval. On assiste de surcroît à une scène carnavalesque (sans nul doute en écho au Buscón) emblématique de la bouffonnerie dans Estebanillo dont l'un des protagonistes est un équidé ${ }^{81}$. Il s'ensuit alors une scène ô combien burlesque de lavement, orchestrée par Estebanillo vêtu comme un médecin et qui fait mine de soigner cet équidé qui finit en fait brûlé par les ventouses qu'on lui pose : "[...] del fuego de la estopa y pelo del jumento se levantaba una grande

70. D’où le jeu de mot quévédien : "[...] era la persona más necesaria de la riña " (op. cit., I, 2, p. 112).

71. Francisco de Quevedo, op. cit., I, 2, p. 111.

72. Ibid. : "con suma majestad, iba a la jineta sobre el dicho pasadizo con pies".

73. Francisco de Quevedo, op. cit., II, 5, p. 206 : «Llevaba un jumento; alquilómele». Don Toribio qualifie pour sa part cette monture de «borrico" (ibid., p. 207).

74. Ibid., p. 206-207.

75. Ibid.

76. Francisco de Quevedo, op. cit., II, 6, p. 214.

77. Estebanillo González, op. cit., I, 1, p. 33.

78. Ibid., p. 45.

79. Ibid., I, 6, p. 295.

80. Ibid.

81. Ibid., II, 8, p. 133. 
bumareda y olor de chamusquina $»^{82}$. Ainsi voit-on Estebanillo faire ressusciter son moribond de mascarade, dans le même temps humanisé car qualifié de "señor burro $\|^{83}$ ou "señor pollino ${ }^{84}$. Comme un Lazare qui ôte ses bandelettes et la toile qui le couvre, la rosse se libère de ses liens dans d'ultimes ruades en blessant Estebanillo, qui à son tour se retrouve dans un lit-tombeau ${ }^{85}$, en une métamorphose continuée. Plus question ici de gloire de Dieu, mais simplement de glorification de courtisans...

La présence d'une rosse conclut déjà en quelque sorte le premier volume d'Estebanillo González en soulignant la lâcheté d'Estebanillo sur les champs de bataille. La peau d'un équidé lui sert en effet de " couverture " au double sens du terme dans une sorte de tombeau de la survie. Ce n'est pas le picaro descendant de Lazare qui est en décomposition, mais cet animal : " [...] habiéndome tendido en tierra aunque vuéltole la cara por el mal olor [...] me eché por colcha el descarnado babieca; y aun no atreviéndome a soltar el aliento lo tuve más de dos horas a cuestas, contento de que pasando plaza de caballo se salvaría el rey de los marmitones $"{ }^{86}$. De façon renouvelée, le tombeau donne vie et un camarade, version profane du Christ, vient l'en sortir. Il convient de surcroît de noter les inversions antihéroïques : Estebanillo se dit "rey de los marmitones " ${ }^{87}$, il prénomme la rosse en décomposition du nom de l'illustre monture du Cid, "babieca ${ }^{88}$, et celle-ci est décrite comme : "centauro al revés " ${ }^{89}$, " hipógrifo $~^{90}$ et " antigua armadura de güesos "11. Or, au début de l'ouvrage, Estebanillo se compare à un centaure. D'où ici un déplacement jusque dans la dépouille de la rosse, comme si une partie du protagoniste était morte. Ainsi, ce dernier pourra renaître...

Il convient sans nul doute de rechercher dans ces attitudes une cause possible de la disparition du désir d'élévation sociale. L'utopie de meilleure situation sociale se meurt parce qu'il n'y a plus de confiance en la nature humaine. En cela Estebanillo González se présente bien comme une ouvre moderne dont le protagoniste nous propose une nouvelle devise : "Mi gusto es mi honra y ande yo caliente y ríase la gente; pues poco importa que mi padre se llame hogaza si yo me muero de hambre $"{ }^{92}$.

Estebanillo apparaît désormais affublé d'une peau de bête à corne comme un satyre ${ }^{93}$, suite à un acte de désobéissance : «que me clavasen en la delantera del peto, como lanzas en ristre, los cuernos del difunto ciervo [...] que del pellejo se

82. Ibid., p. 135

83. Ibid., p. 136.

84. Ibid., p. 138.

85. Ibid., p. 135-136.

86. Ibid., I, 6, p. 308.

87. Ibid.

88. Ibid. On conserve le « $\mathrm{b}$ » minuscule de l'édition utilisée.

89. Ibid.

90. Ibid. L'hippogriffe est un animal fabuleux des romans de chevalerie.

91. Ibid., I, 6, p. 309.

92. Ibid., II, 7, p. 50.

93. Ibid., p. 78 : «[...] no siendo de sátiro ni fauno era trasunto del mismo Barrabás». 
me hiciera una capellina de armas que cubriendo la cabeza sirviese de loriga [...] me vistieron de animal selvático ${ }^{94}$ - et ceci à sa plus grande honte bien qu'il soit prénommé " gentilhomme " : Yo estaba tan avergonzado de verme gentilhombre de Cervera [...] ${ }^{95}$. Cet affront est suivi d'un baptême où est convié son maître mais où, entendons-le de façon symbolique, Estebanillo ne se rend pas.

Notons qu'une autre image de métamorphose est proposée dans cet ouvrage, aussi peu valorisante et liée on l'a vu à l'eau et à l'âne, à savoir celle du serpent qui mue, lorsque son maître Piccolomini essaye de l'obliger à être courageux sur un champ de bataille: "[...] por probar mi valor, aunque ya tenía harta noticia dél, me llevó una manaña consigo, más forzado que de voluntad, diciéndome que me queria hacer un valiente soldado, siendo cosa irremediable si no es quitándome el pellejo como a culebra y volviéndome a hacer de nuevo $"{ }^{96}$. Voici encore une métamorphose qui ne fonctionne pas et où le picaro, homme-cervidé monté à cheval, ressemble fort à un monstre. Et ces figures paraissent d'autant plus monstrueuses quand l'âne est en fait mulet! En somme, comme le dit Pierre Brunet : «[...] la métamorphose aboutit moins à la création d'un animal qu'à la formation d'un "monstre" $"{ }^{97}$.

En définitive, les récits picaresques, avant que le thème de la métamorphose ne se généralise en dehors de la poésie et devienne un principe poétique dans divers romans et nouvelles selon un nouveau mode de l'affleurement mythique, soulignent combien le recours à ce thème revient à dénoncer un manque d'unité - ici plus social que de l'être humain - et dévoile une espérance en un (des) devenir(s) potentiel(s). Cela passe par un discours sur le corps et du corps, car l'alternative est déjà de parler avec son corps. En se métamorphosant, le picaro parle avec son corps et pas seulement sa voix, lui qui avait déjà eu recours à la transgressive première personne du singulier.

Assurément, ces récits ne se libèrent point du corps physique (pas de palingénésie donc) sans pour autant renoncer à une possible métamorphose (et renaissance) dans le corps social. En effet, la métamorphose n'est-elle pas une sorte de fantasme de l'abandon du corps ? On pourrait également se demander si la véritable métamorphose n'est pas celle du regard de l'Autre qui fonde l'identitée ${ }^{98} \ldots$

Il importe néanmoins de noter que les métamorphoses ichtyologiques et asines proposées dans les récits picaresques, à la fois reliées entre elles par la présence liquide ou l'image de la mue du serpent, ne sont pas définitives - et ne permettent pas d'assister à des retours véritables à la situation initiale -, et donc que les auteurs de ces récits leur ôtent par là même une grande part

94. Ibid., p. 75.

95. Ibid., p. 77.

96. Ibid., II, 9, p. 195.

97. Pierre Brunel, op. cit., p. 163.

98. Guy Belzane, op. cit., p. 14 : «Et si on ne se métamorphosait jamais qu’en soi-même? Si la Métamorphose n'était que la réponse, mais ambiguë, mouvante, inquiétante, à l'éternelle question de l'identité ». 
de leur caractère inquiétant. Il n'empêche qu'en développant l'idée centrale de l'interpénétration des mondes, le motif de la métamorphose s'oppose à la notion de fermeture en récusant l'idée de rigidité d'un monde aux formes fixes. C'est aussi une façon de revendiquer la supériorité du rêve (sur la réalité) : "La métamorphose apparaît comme la solution phantasmatique apportée par le "rêveur" à une situation vécue comme impossible, à une insatisfaction essentielle liée à la perception de contradictions "invivables" " ".

Grâce au développement d'une poétique de la métamorphose dans les récits picaresques est suggérée l'idée qu'autre chose est possible. Il convient également de rappeler que cet attrait pour la métamorphose s'accompagne d'une ontologie qui associe le changement à un défaut d'être. Le changement est souvent perçu comme un signe d'imperfection, de mortalité. Il n'empêche que la pensée et la pratique du changement (la sensibilité métamorphique) sont constitutives de l'humanisme. La métamorphose est aussi liée à diverses traditions (comme l'art des alchimistes) et croyances qui nous rappellent qu'elle s'enracine dans le mystère de la genèse du monde - et des mythes - et dans le devenir de l'homme régénéré. Or, l'on n'assiste pas pour les Lazare et leurs descendants à des métamorphoses cycliques comme celles des romans de transformations comme dans le Crotalón de Cristóbal de Villalón ${ }^{100}$.

Il convient également de rappeler qu'à la différence de la conception antique où la métamorphose était ressentie comme positive, le regard chrétien envisage la métamorphose plutôt comme une intervention contre le plan divin, d'où des images liées à la monstruosité, seul Dieu étant à même de créer. On comprend mieux alors comment changer d'apparence peut convoquer d'emblée une perversion des hiérarchies sociales, notamment dans le contexte de la société des refus de l'Espagne classique. Ainsi relève-t-on l'association entre l'idée de métamorphose et celle de "conversion " dans la Seconde partie du Lazarillo (1555) où il est explicitement indiqué : " [...] mirándome a una parte y a otra, por ver si vería en mi alguna cosa que no estuviese convertida en atún, estándome en la cueva muy a mi placer [...] mas hube miedo me conociesen y les fuese manifiesta mi conversión $[\ldots]{ }^{101}$. Sous cet éclairage, la lutte des picaros contre

99. Gisèle Mathieu-Castellani, «Le mythe du phénix et la poétique de la métamorphose dans le lyrisme néo-pétrarquiste et baroque ", dans Poétiques de la métamorphose, op. cit., p. 161-183 (p. 170).

100. Le critique Robert H. Williams a même pensé que l'auteur du Crotalón aurait pu être celui de la seconde partie du Lazarillo de 1555, "Notes on the Anonymous Continuation of Lazarillo de Tormes ", The Romanic Review, 1925, n 16, p. 223-235. Pour une étude précise de cet auteur, se reporter à Marcel Bataillon, Erasmo y España. Estudios sobre la historia espiritual del siglo XVI, México-Madrid-Buenos Aires, Fondo de Cultura Económica, 1986 (1950). (Érasme et l'Espagne, Paris, Droz, 1937), p. 654-668 : "El "Crotalón” y la corriente lucianesca ». Marcel Bataillon présente alors le Crotalón comme un monstre ayant quelques traits communs avec Lazarillo et sa postérité. Il souligne également l'existence d'une relation thématique étroite avec le Diálogo de las transformaciones, où un coq raconte ses aventures à l'époque où il était un âne. Menéndez y Pelayo publia ce texte dans le second tome de ses Orígenes de la novela, Santander, CSIC, 1943 (1905-1910), 4 vols.

101. Segunda Parte de Lazarillo (1555), op. cit., chap. II, p. 26. On choisit de souligner. 
la mort (ici sociale et religieuse), à partir notamment de diverses inversions, paraît d'autant plus utopique. Ces gueux restent picaros et convertis, hybrides marginaux d'un entre-deux, et non pas centres reconnus dans cette dynamique d'évolution. En conséquence, vu que l'on n'assiste pas à la phase finale d'une métamorphose véritable, ne serait-ce que parce que celle-ci ne dure pas, on en reste au niveau de l'utopie du changement possible.

En retournant par la suite dans la caverne, mandaté officiellement par les thons pour tuer le monstre qui s'y trouve, c'est lui-même que Lazare tue symboliquement. Et il doit encore tuer de nombreux thons, entrés trop nombreux dans la grotte sous-marine pour se rendre compte de son exploit, pour éviter l'étouffement et sauver cette fois-ci réellement sa vie, soit un entremêlement de morts et de renaissances. En mangeant le corps des thons tués, il participe davantage à ce cycle de la renaissance. Notons l'humour de cette scène : " [...] haciéndome nuevo de aquel manjar que ya le habia comido algunas veces en Toledo, mas no tan fresco como allí comía ", Lazarillo (1555), op. cit., chap. II, p. 28. 